\title{
INTERSECTION THEORY IN AN EQUICHARACTERISTIC REGULAR LOCAL RING AND THE RELATIVE INTERSECTION THEORY
}

\author{
JAMES HORNELL
}

\begin{abstract}
Using the intersection theory of the notes of Serre, Algèbre locale. Multiplicités, the valuation theoretic formula for a hypersurface is given, and it is shown that transversality is equivalent to intersection multiplicity one. The intersection multiplicity of Algèbre locale. Multiplicités is computed for two algebraic varieties over an arbitrary field and compared to the intersection number of Weil's Foundations of algebraic geometry.
\end{abstract}

The intersection theory and the notation developed in the notes of Serre, Algèbre locale. Multiplicités [3], will be used. Let $A$ be a regular noetherian ring which is locally equicharacteristic. Let $Z$ be the group of cycles of $A$, the free abelian group generated by the prime ideals of $A$. If $p$ is a prime ideal of $A, p$ also denotes the cycle. If $M$ is a noetherian $A$ module and if $p_{1}, \cdots, p_{s}$ are the isolated prime ideals of $M$, let $Z(M)=$ $\sum_{i=1, \ldots, s} \ell_{A_{p_{i}}}(M) p_{i} \in Z$. If $p$ and $q$ are two prime ideals of $A$ which intersect properly at another prime ideal $m$ of $A$, that is $p+q \subset m$ and height $p A_{m}+$ height $q A_{m}=$ height $(p+q) A_{m}=$ height $m A_{m}$, define as in [3, Chapter V], $i(p \cdot q, m)=\chi^{A_{m}}\left(A_{m} / p A_{m}, A_{m} / q A_{m}\right)$. Extending $i$ bilinearly to cycles which intersect properly, $i(Z(M) \cdot Z(N), m)=\chi^{A_{m}}\left(M_{m}, N_{m}\right)$.

Let $p_{1}, \cdots, p_{s}$ be distinct prime ideals of $A$, let $q_{1}, \cdots, q_{t}$ be distinct prime ideals of $A$, and let $a_{i}$ and $b_{j}$ be nonzero integers for $i=1, \cdots, s$ and $j=1, \cdots, t$. The cycles $a_{1} p_{1}+\cdots+a_{s} p_{s}$ and $b_{1} q_{1}+\cdots+b_{t} q_{t}$ intersect properly if for each pair $i, j$ the prime ideals $p_{i}$ and $q_{j}$ intersect properly at each isolated prime ideal $m_{i j k}$ of $p_{i}+q_{j}$. If this holds, define

$$
\left(a_{1} p_{1}+\cdots+a_{s} p_{s}\right) \cdot\left(b_{1} q_{1}+\cdots+b_{t} q_{t}\right)=\sum_{i j k} a_{i} b_{j} i\left(p_{i} \cdot q_{j}, m_{i j k}\right) m_{i j k}
$$

which is again a cycle. Associativity and the projection formula are given in $[3$, Chapter V, C].

Received by the editors June 25, 1971 and, in revised form, December 21, 1971.

AMS 1970 subject classifications. Primary 13H15, 14C15.

Key words and phrases. Relative intersection theory, intersection theory, equicharacteristic regular local ring, transversality, intersection multiplicity one. 
Below are given the valuation theoretic formula for a hypersurface section of a cycle and the equivalence of transversality and intersection multiplicity one. Both follow easily from [3]. A relative intersection number is then defined using the intersection theory of Weil's Foundations of algebraic geometry [4], and it is shown that this intersection number is the intersection multiplicity given by the above theory.

1. The valuation theoretic formula for the intersection of a cycle with a principal cycle. Let $A$ be an equicharacteristic regular local ring with maximal ideal $m$, and let $p$ be a prime ideal of $A$ such that the integral closure of $A / p$ in its quotient field, $I(A / p)$, is a finite $A / p$ module. Let $I(A / p)=(A / p) \alpha_{1}+\cdots+(A / p) \alpha_{n}$. Let $R=A\left[X_{1}, \cdots, X_{n}\right]$, the polynomial ring, which is regular. Let $p^{\prime}$ be the kernel of the homomorphism $h: R \rightarrow$ $I(A / p)$ where $\left.h\right|_{A}$ is the canonical homomorphism onto $A / p$ and $h\left(X_{i}\right)=\alpha_{i}$. The projection $g: \operatorname{Spec} R \rightarrow \operatorname{Spec} A$ induced by $A \subset R$ is proper. Let $m_{1}$, $\cdots, m_{s}$ be the prime ideals of $R$ lying over $m$ which contain $p^{\prime}$.

A principal cycle of $A$ is a cycle of the form $Z(A /(f))$ where $f$ is a nonzero element of $A$. Assume that the prime ideal $p$ and $Z(A /(f))$ intersect properly.

By $[3$, p. V-29]

$$
g_{*}\left(Z\left(R / p^{\prime}\right) \cdot g^{*} Z(A /(f))\right)=g_{*} Z\left(R / p^{\prime}\right) \cdot Z(A /(f)) .
$$

Now, $g^{*} Z(A /(f))=Z(R /(f)), g_{*} Z\left(R / p^{\prime}\right)=\left[Q\left(R / p^{\prime}\right): Q(A / p)\right] p=p$, and $g_{*} m_{i}=\left[R / m_{i}: A / m\right] m$ where $Q S$ denotes the quotient field of an integral domain $S$. By [3, p. V-20, Corollary],

$$
\chi^{R_{m_{i}}}\left(R_{m_{i}} / p^{\prime} R_{m_{i}}, R_{m_{i}} /(f)\right)=\ell_{R_{m_{i}}}\left(\left(R_{m_{i}} / p^{\prime} R_{m_{i}}\right) \otimes_{R_{m_{i}}}\left(R_{m_{i}} /(f)\right)=v_{i}(f)\right.
$$

where $v_{i}$ is the order function of $R_{m_{i}} / p^{\prime} R_{m_{i}}$, the valuation of $R / p^{\prime}$ centered at $m_{i} / p^{\prime}$. Hence $p \cdot Z(A /(f))=g_{*}\left(\sum_{i} v_{i}(f) Z\left(R / m_{i}\right)\right)$ and the desired formula follows,

$$
i(p \cdot Z(A /(f)), m)=\sum_{i}\left[R / m_{i}: A / m\right] v_{i}(f) .
$$

2. The equivalence of transversality and of intersection multiplicity one. Let $A$ be an equicharacteristic regular local ring, let $m$ be its maximal ideal, and let $k$ be the residue field $A / m$. If $p$ is a prime ideal of $A$ let $J(p)$ be the $k$-vector subspace $\left(p+m^{2}\right) / m^{2}$ of $m / m^{2}$. Two prime ideals $p$ and $q$ of $A$ are transversal if and only if $\operatorname{dim}_{k} J(p+q)=$ height $p+$ height $q$.

Proposition 1. Let $p$ and $q$ be two prime ideals of $A$ which intersect properly at $m$. Then $i(p \cdot q, m)=1$ if and only if $p$ and $q$ are transversal. 
Proof. First let $p$ and $q$ be transversal. Then $A / p, A / q$ and $A /(p+q)$ are regular local rings, $A / p$ and $A / q$ are Cohen-Macaulay, and $p+q=m$. By [3, Corollary, p. V-20]

$$
\chi^{A}(A / p, A / q)=\ell_{A}\left(A / p \otimes_{A} A / q\right)=\ell_{A}(A / m)=1 .
$$

Secondly let $i(p \cdot q, m)=1$. By $[3, p$. V-20]

$$
1=\chi^{\Lambda}(A / p, A / q) \geqq \ell_{A}\left(A / p \otimes_{A} A / q\right) \geqq 1 .
$$

So $\ell_{A}\left(A / p \otimes_{A} A / q\right)=1$, but $A /(p+q)$ is artinian, and hence $p+q=m$. Thus $p$ and $q$ are transversal, for $A$ is regular.

3. Intersection theory in algebraic geometry relative to an arbitrary field. Let $\Omega$ be an algebraically closed field, let $A_{n}$ denote the $n$-dimensional affine space with coordinates in $\Omega$ (the set of all $n$-tuples of elements of $\Omega$ ), let $k$ be a subfield of $\Omega$, and let $k_{a}$ be the algebraic closure of $k$ in $\Omega$. Let $U$ and $V$ be two $k$-varieties in $A_{n}$ which intersect properly at a $k$-variety $W$. Let $U=\bigcup U_{i}$ and $V=\bigcup V_{j}$ where $U_{i}$ and $V_{j}$ are the distinct $k_{a^{-}}$ components of $U$ and $V$ respectively. Let $W_{1}$ be a $k_{a}$-component of $W$. Define an intersection multiplicity relative to $k$, by

$$
i(U \cdot V, W)=\frac{[k(U): k]_{\iota}[k(V): k]_{c}}{[k(W): k]_{\iota}} \sum_{i j} i\left(U_{i} \cdot V_{j}, W_{1}\right)
$$

where $i\left(U_{i} \cdot V_{j}, W_{1}\right)$ is the intersection number of Weil's Foundations [4, p. 148], and [K:k] is the degree of inseparability of $K$ over $k$.

If a $k$-variety $V$ is considered to be a $k$-cycle on $A_{n}$, then $V$ is of the form $V=[k(V): k]_{\iota}\left(V_{1}+\cdots+V_{s}\right)$ where $V_{1}, \cdots, V_{s}$ are the distinct $k_{a^{-}}$ components of $V$, and the above intersection multiplicity appears to be correct. Using the language and results of [4], associativity and the projection formula can be proven. Using the mixed jacobian of [5] for the definition of transversality, the equivalence of intersection multiplicity one and of transversality can be proven in this same language.

However it is more expedient to use the intersection theory of [3].

Proposition 2. Let $U$ and $V$ be two varieties in $A_{n}$ intersecting properly at a $k$-variety $W$. Let $\mathcal{O}$ be the local ring of $A_{n}$ at $W$ over $k(\mathcal{O}=$ $\left.\left\{F / G \mid F, G \in k\left[A_{n}\right]=k\left[X_{1}, \cdots, X_{n}\right], G(W) \neq 0\right\}\right)$, let $m$ be its maximal ideal, and let $p$ and $q$ be the prime ideals in $\mathcal{O}$ of $U$ and of $V$ respectively. Then,

$$
i(U \cdot V, W)=i(p \cdot q, m) .
$$

Proof. Let $k^{\prime}$ be a finite field extension of $k$ over which the $k_{a}$-components of $U, V$ and $W$ are regular. Let $\mathcal{O}^{\prime}$ be $\mathcal{O} \otimes_{k} k^{\prime}$ reduced. The map 
$f: \operatorname{Spec} \mathcal{O}^{\prime} \rightarrow \operatorname{Spec} \mathcal{O}$ induced by the inclusion is proper, and by [3, p. V-29], $f_{*}\left(f^{*} p \cdot f^{*} q\right)=\left(f_{*} f^{*} p\right) \cdot q$. Let $p_{1}, \cdots, p_{s}$ and $q_{1}, \cdots, q_{t}$ be the prime ideals of $\mathcal{O}^{\prime}$ lying over $p$ and $q$ respectively. Let $m_{1}, \cdots, m_{r}$ be the prime ideals of $\mathcal{O}^{\prime}$ lying over $m$.

Let $\mathcal{O}_{i}$ denote the localization of $\mathcal{O} \otimes_{k} k^{\prime}$ at $p_{i}$, and let $p_{i}$ also denote the maximal ideal of $\mathcal{O}_{i}$. Then

$$
f^{*} p=\sum_{i=1, \cdots, s} \ell_{\mathcal{O}_{p_{i}}^{\prime}}\left(\mathcal{O} / p \otimes_{\mathcal{O}} \mathcal{O}_{p_{i}}^{\prime}\right) \cdot p_{i}
$$

and

$$
\ell_{\mathcal{O}_{\nu_{i}}^{\prime}}\left(\mathcal{O} / p \otimes_{\mathcal{C}} \mathcal{O}_{p_{i}}^{\prime}\right)=\ell_{\mathcal{C}_{i}}\left(\mathcal{O} / p \otimes_{\mathcal{O}} \mathcal{O}_{i}\right)=\ell_{\mathcal{C}_{i}}\left(K \otimes_{k} k^{\prime}\right)
$$

where $K=\mathcal{O}_{p} / p \mathcal{O}_{p}=k(U)$. Let $L$ be a purely transcendental extension of $k$ with $K$ (finite) algebraic over $L$. $L$ and $k^{\prime}$ are linearly disjoint over $k$, so $L \otimes_{k} k^{\prime}=L k^{\prime}$ and $K \otimes_{k} k^{\prime}=K \otimes_{L} L k^{\prime} . K \otimes_{L} L k^{\prime}$ is artinian, the prime ideals $\mathfrak{p}_{i}$ of $K \otimes_{L} L k^{\prime}$ correspond to the $p_{i}$, and $\mathcal{O}_{i}$ acts on $\left(K \otimes_{L} L k^{\prime}\right)_{\mathfrak{p}_{i}}$ which is the component of $K \otimes_{L} L k^{\prime}$ not contained in $\mathfrak{p}_{i}$. The $p_{i}$ are conjugate over $k$, the $\mathfrak{p}_{i}$ are also conjugate over $k$, and $\left(K \otimes_{L} L k^{\prime}\right) / \mathfrak{p}_{i} \simeq$ $k^{\prime}\left(U_{i}\right) \simeq K k^{\prime}$.

$$
\begin{aligned}
\ell_{\Theta_{i}}\left(K \otimes_{L} L k^{\prime}\right) & =s^{-1} \ell_{K k^{\prime}}\left(K \otimes_{L} L k^{\prime}\right)=s^{-1}\left[K k^{\prime}: L\right]^{-1} \operatorname{dim}_{L}\left(K \otimes_{L} L k^{\prime}\right) \\
& =s^{-1}\left[K k^{\prime}: L\right]^{-1}\left[k^{\prime}: k\right][K: L] \\
& =s^{-1}\left[k^{\prime}\left(U_{i}\right): k(U)\right]^{-1}\left[k^{\prime}: k\right]=[k(U): k]_{\iota},
\end{aligned}
$$

for $s=\left[k^{\prime}: k\right]_{s} /\left[k^{\prime}\left(U_{i}\right): k(U)\right]_{s}$ and $[k(U): k]_{\imath}=\left[k^{\prime}: k\right]_{i} /\left[k^{\prime}\left(U_{i}\right): k(U)\right]_{i}$ by $[4$, Propositions 26,27, p. 23]. Thus $f^{*} p=[k(U): k]_{\imath}\left(p_{1}+\cdots+p_{s}\right)$. Now, $f_{*} m_{i}=\ell_{\mathcal{O}}\left(\mathcal{O}_{m_{i}}^{\prime} / m_{i} \mathcal{O}_{m_{i}}^{\prime}\right) m$, and

$$
\ell_{\mathcal{O}}\left(\mathcal{O}_{m_{i}}^{\prime} / m_{i} \mathcal{O}_{m_{i}}^{\prime}\right)=\ell_{C / m}\left(k^{\prime}\left(W_{1}\right)\right)=\left[k^{\prime}\left(W_{1}\right): k(W)\right]=\left[k: k^{\prime}\right] / r[k(W): k]_{i} .
$$

By the first computation, and a computation similar to the second, $f_{*} f^{*} p=\left[k^{\prime}: k\right] p$. Thus,

and

$$
\left[k^{\prime}: k\right] i(p \cdot q, m)=\frac{\left[k: k^{\prime}\right][k(U): k]_{\iota}[k(V): k]_{\iota}}{r[k(W): k]_{\iota}} \sum_{i j k} i\left(p_{i} \cdot q_{j}, m_{k}\right)
$$

$$
i(p \cdot q, m)=\frac{[k(U): k]_{\iota}[k(V): k]_{\iota}}{[k(W): k]_{\iota}} \sum_{i j} i\left(p_{i} \cdot q_{j}, m_{1}\right) .
$$

If $k^{\prime \prime}$ is a finite algebraic extension of $k^{\prime}$ it follows, by the above, that $\chi^{\mathcal{O}^{\prime}}\left(\mathcal{O}^{\prime}\left|p_{i}, \mathcal{O}^{\prime}\right| q_{j}\right)=\chi^{\mathcal{O}^{\prime \prime}}\left(\mathcal{O}^{\prime \prime}\left|p^{\prime \prime}, \mathcal{O}^{\prime \prime}\right| q^{\prime \prime}\right)$, where $\mathcal{O}^{\prime \prime}=\mathcal{O}^{\prime} \otimes_{k} k^{\prime \prime}, p^{\prime \prime}=p_{i} \otimes_{k} k^{\prime \prime}$, $q^{\prime \prime}=q_{j} \otimes_{k} k^{\prime \prime} \cdot \chi^{A}(M, N)=\sum_{i} \ell_{A}$ Tor $_{i}^{A}(M, n)$ commutes with direct limits, so $\chi \mathcal{U}^{\prime}\left(\mathcal{O}^{\prime}\left|p_{i}, \mathcal{O}^{\prime}\right| q_{j}\right)=i\left(U_{i} \cdot V_{j}, W_{1}\right)$ by $[3, \mathrm{p} . \mathrm{V}-21$, Theorem 1$]$. 


\section{BIBLIOGRAPHY}

1. D. G. Northcott, Lessons on rings, modules and multiplicities, Cambridge Univ. Press, London, 1968. MR 38 \#144.

2. P. Samuel, Methodes d'algèbre abstraite en géométrie algébrique, Ergebnisse der Mathematik und ihrer Grenzgebiete, Heft 4, Springer-Verlag, Berlin, 1955. MR 17, 300.

3. J.-P. Serre, Algèbre locale. Multiplicités, Cours au Collège de France, 1957/58, rédigé par Pierre Gabriel, 2ième éd., Lecture Notes in Math., no. 11, Springer-Verlag, Berlin and New York, 1965. MR 34 \#1352.

4. A. Weil, Foundations of algebraic geometry, Amer. Math. Soc. Collog. Publ., vol. 29, Amer. Math. Soc., Providence, R.I., 1946. MR 9, 303.

5. O. Zariski, The concept of a simple point on an abstract algebraic variety, Trans. Amer. Math. Soc. 62 (1947), 1-52. MR 9, 99.

6. - The fundamental ideas of abstract algebraic geometry, Proc. Internat. Congress Math. (Cambridge, Mass., 1950), vol. 2, Amer. Math. Soc., Providence, R.I., 1952, pp. 77-89. MR 13, 578.

7. O. Zariski and P. Samuel, Commutative algebra. Vols. I, II, University Series in Higher Math., Van Nostrand, Princeton, N.J., 1958, 1960. MR 19, 833; MR 22 \#11006.

Department of Mathematics, University of Kansas, Lawrence, Kansas 66044 